\title{
QUALIDADE DE VIDA DOS USUÁRIOS DA ATENÇÃO PRIMÁRIA À SAÚDE: PERFIL E FATORES QUE INTERFEREM
}

\author{
Márcia Helena Miranda Cardoso PODESTÁ ${ }^{1}$ \\ Walnéia Aparecida de SOUZA ${ }^{2}$ \\ Olinda M. G. C. VILAS BOAS ${ }^{3}$ \\ Aline Daniel MARTINS ${ }^{4}$ \\ Cyntia de Lima BRAZ ${ }^{4}$ \\ Eric Batista FERREIRA ${ }^{5}$
}

1. Professora de Farmacologia da Universidade Federal deAlfenas-MG. Mestre e Doutora em Farmacologia (Unifal-MG/Unicamp).

2. Professora de Farmácia Hospitalar e Assistência Farmacêutica da Universidade Federal de Alfenas. Mestre e Doutora em Farmacologia (Unifal-MG/Unicamp).

3. Professora de Farmacologia e Assistência Farmacêuticada Universidade Federal de Alfenas. Doutora em Farmacologia pela Unicamp.

4. Discentes do curso de Farmácia da UNIFAL-MG.

5. Professor de Estatística da Universidade Federal de Alfenas. Doutor em Estatística da Unifal-MG e Experimentação Agropecuária (UFLA/Open University).

\author{
Autor correspondente: \\ Márcia Helena Miranda Cardoso Podestá \\ Rua Gabriel Monteiro da Silva, 700 \\ Centro, Alfenas-MG e-mail: mhmcardoso@ hotmail.com
}

Recebido em: 29/10/2013 - Aprovado em: 21/12/2013 - Disponibilizado em: 15/01/2014

RESUMO: A análise da qualidade de vida (QV) dos pacientes tem sido um indicador clínico cada vez mais relevante em termos de avaliação de tratamentos, de intervenções e de melhorias da saúde. O objetivo do estudo foi analisar a QV dos pacientes atendidos pela Atenção Primária à Saúde (APS), bem como os fatores interferentes. A pesquisa foi desenvolvida em quatro centros de Atenção Primária do Sistema Único de Saúde do município de Alfenas-MG. Foram incluídos 100 usuários da APS, de ambos os sexos, com idade igual ou superior a 18 anos. Analisaram-se aspectos sociodemográficos, perfil farmacoepidemiológico, hábitos de vida e QV pelo instrumento SF-36. Para a análise estatística, foram utilizados os testes de Scott-Knott, Wilcoxon e Kruskal-Wallis. Dos entrevistados, 68\% são mulheres e 50\% estão na faixa etária de 45 a 65 anos; $69 \%$ não praticam exercícios físicos. Quanto ao perfil farmacoepidemiológico, houve prevalência do uso de anti-hipertensivos (78\%). Em relação à QV, o domínio que se apresentou mais comprometido foi a vitalidade $(69,75 \%)$ e o melhor índice foi encontrado no domínio aspectos sociais (86,27\%). Dentre as Unidades de Saúde, o PSF 4 foi o que apresentou os domínios da QV mais baixos, sendo o único situado na periferia. Os fatores que interferiram na QV foram o gênero, a polifarmácia e a faixa etária. Neste estudo, constatou-se que os pacientes atendidos nas UAPS apresentam, em geral, boa qualidade de vida, que varia de acordo com fatores como gênero, a idade, a o uso de polifarmácia e o fator socioeconômico.

Palavras-chave: Atenção Primária à Saúde; Qualidade de Vida. SF-36.

ABSTRACT: The analysis of quality of life (QOL) of patients has been a clinical indicator increasingly important in terms of evaluating treatments, interventions and health improvements. The aim of the study was to assess the QOL of patients seen by the Primary Health Care (PHC), as well as check that interfering factors. The research was conducted 
in four health centers in primary care Health System in the city of Alfenas, MG. We included 100 users of the PHC, of both sexes, aged 18 years and over. We analyzed sociodemographic, pharmacoepidemiological profile, lifestyle and QOL by SF-36 instrument. For statistical analysis, we used the tests of Scott-Knott, Wilcoxon and Kruskal-Wallis. Of the respondents, $68 \%$ are women and $50 \%$ are aged 45 to 65 years, $69 \%$ did not practice any physical exercise. As the profile pharmacoepidemiological was prevalent use of antihypertensive drugs (78\%). Regarding QOL, the domain that was most affected was the vitality (69.75\%) and the highest rate was found in the social aspects domain (86.27\%). Among the Health Units, the FHP 4 showed the lowest domains of QOL, being the only located in the periphery. The factors that interfere in QOL were gender, polypharmacy and age. In this study, it was found that patients treated at Health Units, have generally good quality of life, which varies according to factors such as gender, age, use of polypharmacy and socioeconomic factors.

Key words: Public Health System. Quality of life. SF-36

\section{INTRODUÇÃO}

De acordo com a Organização Mundial de Saúde (OMS), a qualidade de vida (QV) é definida como “a percepção do indivíduo de sua posição na vida, no contexto da cultura e sistema de valores nos quais vive e em relação aos seus objetivos, expectativas, padrões e preocupações". A análise da QV dos pacientes tem sido um indicador clínico cada vez mais relevante em termos de avaliação de tratamentos, de intervenções e de melhorias da saúde.

Ante essa preocupação, o número de instrumentos para a avaliação da QV vem crescendo de forma acelerada desde meados do século XX. Dentre esses instrumentos, surgiu o Medical Outcomes Study 36 - Item Short-Form Health Survey (SF-36), já validado no Brasil (CICONELLI, 1997; SEIDL; ZANNON, 2004). Desde então, observa-se que na última década muitos pesquisadores brasileiros têm se preocupado em mapear a QV dos pacientes utilizando o SF-36, em diversas condições clínicas (SOARES, et al., 2008; TRAVASSOS et al.,
2002; NOGUEIRA, et al., 2003; CASTRO, et al., 2003; MIRANDA, 2010) .

Com a Constituição de 1988, o Ministério da Saúde assumiu o compromisso de reestruturar o modelo de atenção à saúde no Brasil, partindo de um referencial de saúde como direito de cidadania, pressupondo a organização de serviços cada vez mais resolutivos, integrais e humanizados. Nessa proposta, o poder público municipal fica investido da responsabilidade imediata de atendimento das necessidades e das demandas de saúde de todos os seus munícipes, contando com a devida cooperação técnica e financeira da União e dos estados, de acordo com o previsto na Norma Operacional Básica do Sistema Único de Saúde (BRASIL, 1996). Essa estratégia de descentralização objetiva facilitar o acesso dos indivíduos e possibilitar uma gerência de saúde mais adequada ao contexto da população atendida, oferecendo serviços de melhor qualidade. No âmbito dessas mudanças, várias políticas municipais têm se organizado a partir do Programa de Saúde da Família (PSF), proposta que se insere no nível da atenção básica e que 
apresenta como objetivo final promover a QV e o bem-estar individual e coletivo por meio de ações e serviços de promoção, de proteção e de recuperação da saúde (SOUZA; CARVALHO, 2003).

Diante do exposto, observa-se que a QV constitui-se em um dos parâmetros essenciais dentro do sistema de saúde. Sabendo-se que as Unidades de atenção primária à saúde (UAPS) constituem a porta de entrada dos pacientes no sistema de saúde, e que este nível de atenção deve garantir resolução de cerca de $80 \%$ das necessidades e dos problemas de saúde da população de um município (BRASIL, 2004), torna-se fundamental avaliar a QV dos usuários da UAPS. Portanto, este instrumento é fundamental para o planejamento e para possíveis adequações das UAPS, que constitui parte essencial e promissora do sistema de saúde, visto que a demanda tende a aumentar nas próximas décadas em decorrência do rápido processo de envelhecimento populacional brasileiro. Assim, o objetivo do presente estudo foi analisar a QV dos usuários das UAPS e verificar quais os fatores interferentes.

\section{METODOLOGIA}

Este estudo seguiu as recomendações da Resolução 196/96 do Conselho Nacional de Saúde, e foi aprovado pelo comitê de ética em Pesquisa da Universidade Federal de
Alfenas-MG (UNIFAL-MG) sob o número 23087.003008/2009-67.

\section{Local do Estudo}

O presente estudo foi realizado no município de Alfenas-MG, que possui 73.722 habitantes (IBGE, 2010). Atualmente, o município conta com 13 equipes de Programa de Saúde da Família (PSF) e quatro Unidades Básicas de Saúde (UBS), o que representa em torno de $60 \%$ de cobertura populacional.

\section{População de estudo}

Foram entrevistados 100 usuários atendidos nas UAPS, de ambos os sexos, com idade igual ou superior a 18 anos, sendo aplicados 25 formulários em cada Unidade de Saúde. O cálculo do tamanho da amostra foi realizado por amostragem aleatória por conglomerado e os locais para a realização das entrevistas foram definidos por sorteio. Com isso, foram estudadas 4 UAPS doravante denominadas 1, 2, 3 e 4 . As três primeiras apresentam um perfil semelhante em relação à população, lotadas em regiões mais centrais, diferentes da unidade 4 que se encontra em uma região periférica e de maior precariedade.

A coleta de dados foi realizada durante os meses de agosto e setembro de 2009. O convite aos usuários foi realizado no momento em que aguardavam a consulta no ambulatório ou logo após o término da mesma. 


\section{Instrumento para a coleta de dados}

Para a obtenção dos dados, foram utilizados questionários a fim de avaliar as características sociodemográficas, os hábitos de vida, a presença de morbidades e o perfil farmacoepidemiológico.

Para a análise da QV, utilizou-se o Medical Outcomes Study 36 - Item ShortForm Health Survey (SF-36), instrumento genérico criado por Ware e Sherbourne em 1992 e por Ware e colaboradores em 1992, a partir do Medial Outcome Study (MOS), para avaliar estado de saúde. O SF-36, traduzido e validado para o português por Ciconelli (1997), é um instrumento de verificação de característica genérica, ou seja, pode ser utilizado em qualquer situação de agravo à saúde. Esse instrumento é composto de 36 itens, agregados em 8 dimensões ou domínios o qual investiga aspectos distintos: capacidade funcional; aspectos físicos; dor; estado geral de saúde; vitalidade; aspectos sociais; aspectos emocionais e saúde mental. Prevê escores de 0 a 100 para cada dimensão (SOARES, et al., 2010).

\section{Análise dos dados:}

A QV foi escolhida como variável dependente, correlacionada com as seguintes variáveis independentes: localização da das UAPS; gênero; uso de polifarmácia (uso de cinco ou mais medicamentos) e idade. Para a comparação entre as pontuações das diferentes Unidades Básicas de Saúde de
Alfenas, os dados do SF-36 foram submetidos à análise de variância em delineamento em blocos casualizados (DBC), seguida pelo teste de Scott-Knott, ambos a 5\% de significância. Nela, foram comparados os bairros e as dimensões foram consideradas blocos. $\mathrm{Na}$ comparação entre as pontuações geradas pelo SF-36 referentes ao gênero e à polifarmácia, foi aplicado o teste de Wilcoxon a 5\% de significância. Também foi realizado o teste de Kruskal-Wallis a 5\% de significância para verificar a igualdade entre as pontuações obtidas nas faixas etárias dos entrevistados. As análises estatísticas foram realizadas por meio do software $\mathrm{R}$ ( $\mathrm{R}$ DEVELOPMENT CORE TEAM, 2011), do qual foi utilizada a função $d b c$ (FERREIRA, 2009) do pacote ExpDes: Experimental Designs (CAVALCANTE, 2007).

\section{RESULTADOS}

\section{Caracterização Sociodemográfica:}

A maioria dos entrevistados pertence ao gênero feminino (68\%); de raça branca $(82 \%)$ e com baixa renda familiar (62\%) e escolaridade (63\%); com a idade variando de 18 a 70 anos, sendo mais expressiva a faixa etária de 45 a 65 anos (49\%).

\section{Hábitos de vida e morbidades:}

Em relação aos hábitos de vida, os resultados estão demonstrados na Tabela 1 . Quanto à morbidade, os tipos de patologias 
mais freqüentemente encontrados na população pesquisada também estão apresentados na mesma tabela; verificou-se que $45 \%$ dos pacientes são portadores de hipertensão.

Tabela 1 - Perfil de cuidados gerais de saúde, hábitos de vida e morbidades dos usuários atendidos nas UAPS de Alfenas-MG.

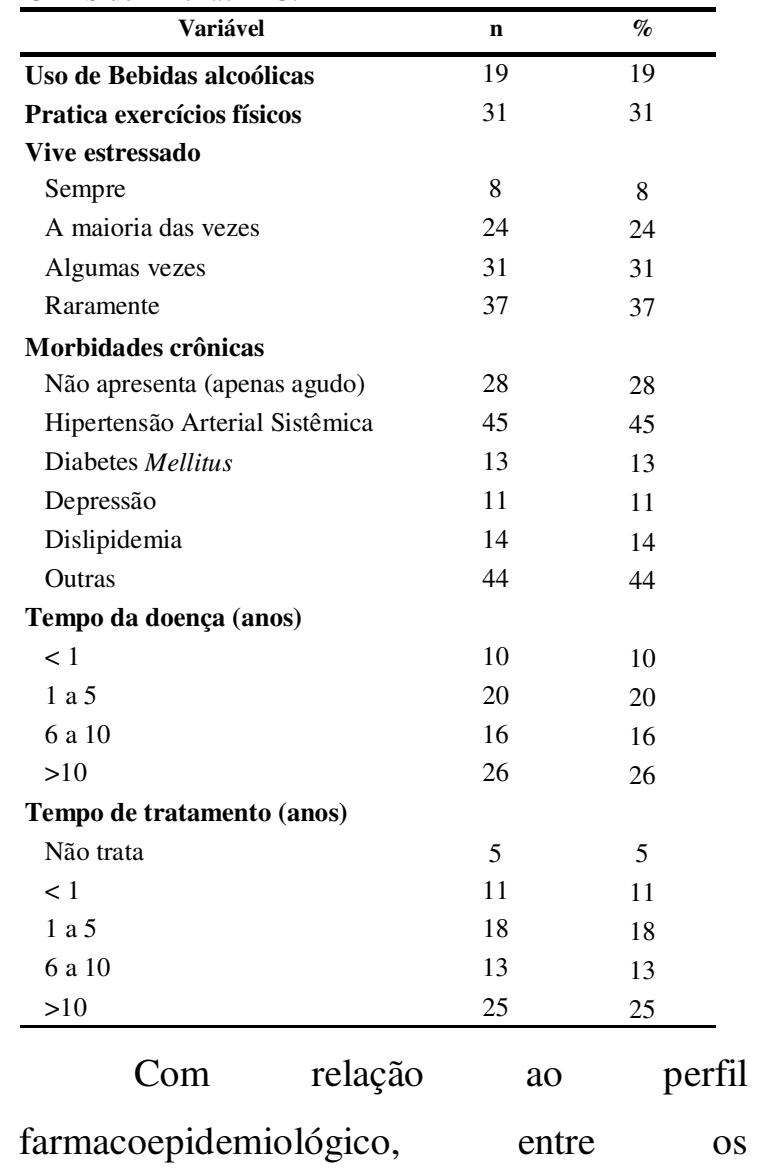

entrevistados que faziam uso de medicamentos, houve prevalência do uso de anti-hipertensivos (78\%); de hipoglicemiantes (22\%); de protetores gástricos (13\%); de antidepressivos (9\%); hipolipemiantes (6\%) e de outros (47\%) (Figura 1).

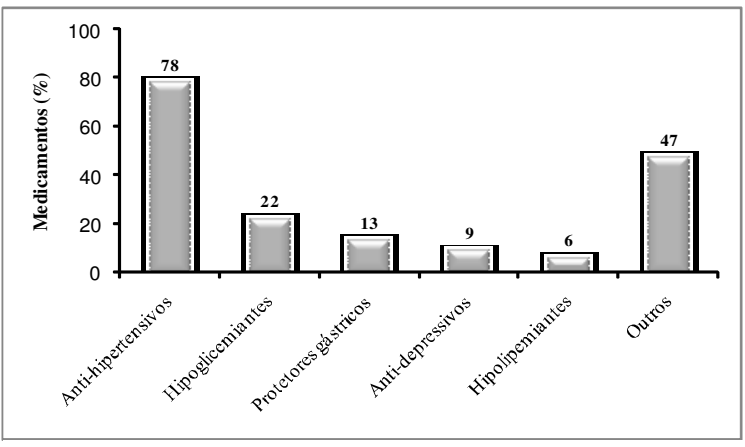

Figura 1 - Principais classes de medica mentos utilizados pelos entrevistados.

\section{Qualidade de vida}

Com relação à avaliação geral da QV, a dimensão que apresentou maior comprometimento foi a vitalidade, enquanto que o melhor índice foi obtido com o domínio aspectos sociais. Já os aspectos físicos, de dor e de estado geral de saúde apresentaram semelhança nos scores (Figura 2).

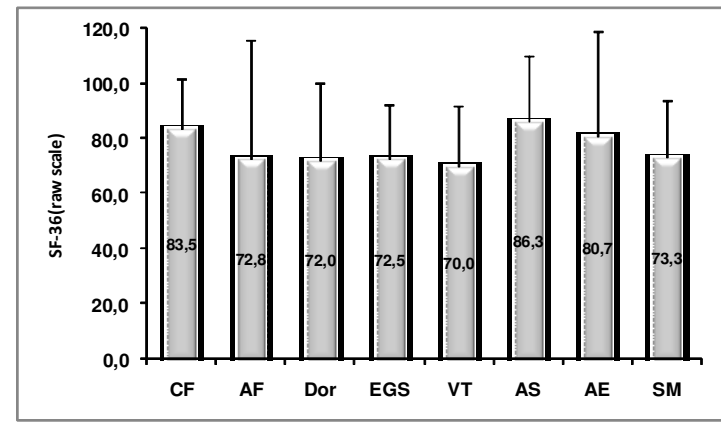

Figura 2 - Qualidade de vida, segundo os domínios do SF-36, dos pacientes atendidos nas UAP S em Alfenas-MG. CF- capacidade funcional; AF - aspecto físicos; EGS - estado geral de saúde; VT - vitalidade; AS - a spectos socia is; AE aspectos emocionais, SM- saúde mental.

Analisando-se a QV em cada unidade de saúde estudada, verificou-se, por meio do teste de análise de variância a $5 \%$ de significância, que a maioria dos scores de QV dos pacientes das unidades 1,2 e 3 foram estatisticamente semelhantes, com resultado diferente apenas para a dimensão dor. Para esta, apenas a unidade 3 apresentou um valor estatisticamente diferente, superior aos 
Tabela 2 - Qualidade de vida (SF-36), em cada Unidade de Saúde, dos usuários atendidos nas UAPS de Alfenas-MG.

\begin{tabular}{lcccc}
\hline \multicolumn{1}{c}{ Dimensões } & Unidade 1 & Unidade 2 & Unidade 3 & Unidade 4 \\
\cline { 2 - 5 } do SF-36 & Media \pm DPM & Media \pm DPM & Media \pm DPM & Media \pm DPM \\
\hline Capacidade funcional & $85,4(17,1)$ & $84,8(16,6)$ & $89,20(18,1)$ & $74,6(19,5)$ \\
Aspectos físicos & $72,0(44,1)$ & $79,0(36,6)$ & $85,00(35,4)$ & $\mathbf{5 5 , 0 * ( 5 0 )}$ \\
Dor & $69,7(29,1)$ & $69,5(25,4)$ & $\mathbf{8 5 , 7} *(\mathbf{2 5 , 3 )}$ & $63,6(28,1)$ \\
Estado Geral de Saúde & $76,8(20,5)$ & $68,4(21,8)$ & $73,3(16,2)$ & $71,6(20,9)$ \\
Vitalidade & $69,8(20,9)$ & $63,0(22,0)$ & $72,40(22,9)$ & $73,8(20,4)$ \\
Aspectos sociais & $85,0(23,9)$ & $89,5(18,3)$ & $91,50(22,4)$ & $79,0(27,7)$ \\
Aspectos emocionais & $81,3(38,6)$ & $88,0(27,0)$ & $89,3(30)$ & $\mathbf{6 4 , 0 * ( 4 8 , 9 )}$ \\
Saúde Mental & $77,1(17,9)$ & $66,1(22,5)$ & $77,3(20,3)$ & $72,6(20,1)$ \\
\hline
\end{tabular}

Médias seguidas da mesma letra não diferem entre si de acordo com o teste de Scott-Knott a

$5 \%$ de significância, dentro de cada linha.

demais. Já a unidade 4 apresentou valores inferiores aos demais nos domínios aspectos físicos e aspectos emocionais. Os dados estão apresentados na Tabela 2 .

Estratificando-se a população por gênero, observou-se diferença estatisticamente significativa em dois domínios do SF-36, sendo que a QV apresentada pelos homens foi inferior à obtida pelas mulheres nos domínios vitalidade ( $\mathrm{p}=0,0035)$ e saúde mental $(\mathrm{p}=0,00001)$ (teste de Wilcoxon a $5 \%$ de significância) (Figura $3)$.

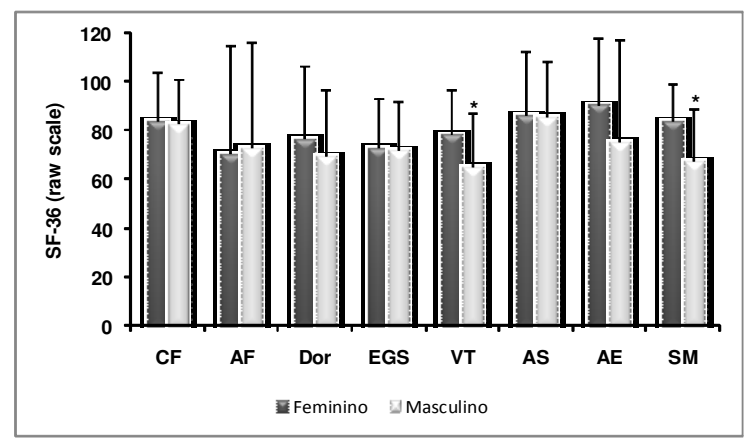

Figura 3 - Valores obtidos para cada domínio do SF- 36 por sexo (feminino $\mathrm{n}=68 \mathrm{e}$ masculino $n=32$ ). * teste de Wilcoxon a $5 \%$ de significância.

Com relação ao uso de medicamentos, os pacientes que fazem uso de polifarmácia ( 5 medicamentos ou mais), apresentaram pontuações inferiores; mas somente os domínios capacidade funcional $(\mathrm{p}=0,0249)$, estado geral de saúde $(\mathrm{p}=0,0004)$ e vitalidade $(\mathrm{p}=0,0125)$ foram os que apresentaram diferenças estatisticamente significativas (teste de Wilcoxon a $5 \%$ de significância). Os dados estão apresentados na Figura 4.

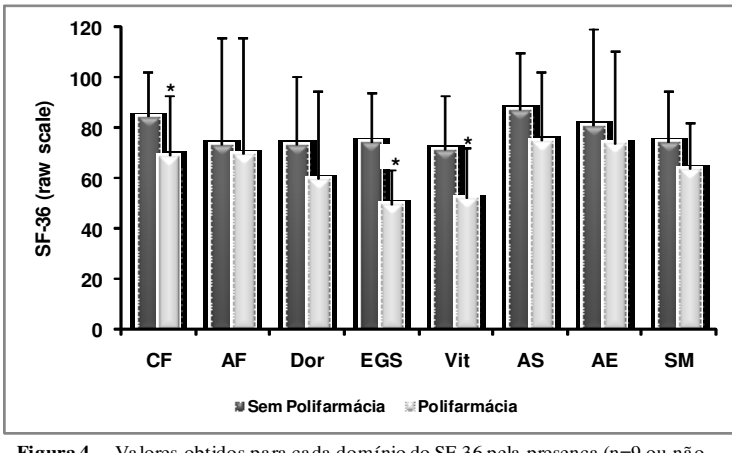

Figura 4 - Valores obtidos para cada domínio do SF-36 pela presença (n=9 ou não $\mathrm{n}=91$ ) de polifarmácia. $*$ teste de Wilcoxon a 5\% de significância.

Ao comparar as faixas etárias, observou-se que os domínios capacidade funcional $(\mathrm{p}=0,0037)$ e aspectos sociais $(\mathrm{p}=$ 0,0407) dos pacientes acima de 65 anos foram estatisticamente inferiores às outras faixas etárias. Em relação ao domínio estado geral de saúde, a faixa etária de 45 a 65 anos 
Tabela 3 - Valores obtidos para cada domínio do SF-36 por faixa etária dos usuários atendidos nas UAPS de Alfenas-MG.

\begin{tabular}{lcccc}
\hline \multicolumn{1}{c}{$\begin{array}{c}\text { Dimensões } \\
\text { do SF-36 }\end{array}$} & $\mathbf{1 8}$ a $<\mathbf{3 0}$ anos & $\mathbf{3 0}$ a $<\mathbf{4 5}$ anos & $\mathbf{4 5}$ a 65 anos & $>\mathbf{6 5}$ anos \\
\cline { 2 - 5 } & Media \pm DPM & Media \pm DPM & Media \pm DPM & Media \pm DPM \\
\hline Capacidade funcional & $94,5(9,26)$ & $90,4(12,3)$ & $80,71(18,6)$ & $\mathbf{7 4 , 3}(23,5)^{*}$ \\
Aspectos físicos & $92,5(23,7)$ & $80(36,8)$ & $68,37(45,3)$ & $62,5(50,0)$ \\
Dor & $79,7(29,1)$ & $74,6(28,4)$ & $68,47(28,3)$ & $74,69(26,8)$ \\
Estado Geral de Saúde & $79,3(19,9)$ & $78,44(22,6)$ & $\mathbf{6 8 , 2}(17,9) *$ & $72,25(19,7)$ \\
Vitalidade & $67(21,1)$ & $71,6(19,3)$ & $70(23,5)$ & $67,81(21,2)$ \\
Aspectos sociais & $95(10,5)$ & $94,5(14,0)$ & $80,1(28,5)$ & $\mathbf{8 6 , 7}(19,1)^{*}$ \\
Aspectos emocionais & $83,33(36)$ & $84(37,4)$ & $76,19(41,4)$ & $87,5(29,5)$ \\
Saúde Mental & $76,4(16,3)$ & $72(24,9)$ & $72,08(29,2)$ & $77,16(16,6)$ \\
\hline
\end{tabular}

DP: Desvio Padrão; * teste de Wilcoxon a 5\% de significância.

apresentou valor inferior à de maiores que 65

apresentados

na

Tabela

3.

anos $(\mathrm{p}$-valor $=0,001)$. Os resultados estão

\section{DISCUSSÃO}

Nas últimas décadas, os resultados da atenção à saúde têm sido analisados, valorizando, cada vez mais, à ótica do paciente. Os benefícios de tratamentos específicos e dos sistemas de assistência à saúde em geral vêm sendo avaliados, considerando-se a extensão da mudança no estado funcional e no bem-estar do indivíduo, à luz de suas necessidades e expectativas (HALFOUN; MATTOS; AGUIAR, 2007).

Dos 100 usuários entrevistados, a maioria era do sexo feminino. A maior utilização dos serviços de saúde pelas mulheres é comum a outros estudos brasileiros dos usuários do SUS. Esse dado pode ser explicado por questões relacionadas à saúde reprodutiva e pelo fato de que as mulheres tendem a se preocupar mais com sua

própria saúde do que os homens (RIBEIRO et al., 2006).

A maioria dos indivíduos relatou baixa escolaridade e baixa renda familiar, justificando o atendimento pelo sistema público dos grupos com inserção social mais precária, cumprindo as expectativas relativas ao desempenho dessa política pública.

$$
\text { A patologia crônica mais }
$$
frequentemente encontrada na população estudada foi a hipertensão. Estes dados são compatíveis com a característica de atendimento das unidades de saúde onde os dados foram coletados. Em todos os serviços funcionam Ligas de Hipertensão e Diabetes, razão pela qual estes foram os tipos de afecções mais encontrados (45 \% e 13\%, respectivamente). 
Com relação a classe de medicamentos, os anti-hipertensivos foram os mais utilizados $(78 \%)$. O grande número de medicamentos desta classe se justifica, uma vez que é comum a terapêutica combinada de anti-hipertensivos. De acordo com a VI DIRETRIZES BRASILEIRAS DE HIPERTENSÃO, vários estudos mostram que em cerca de 2/3 dos casos, a monoterapia não foi suficiente para atingir as reduções de pressão previstas, havendo clara tendência atual para a introdução de terapêutica combinada de anti-hipertensivos.

Dentre os que entrevistados que apresentaram alguma morbidade, verificou-se que a maioria realiza o tratamento desde o diagnóstico médico, uma vez que o tempo de tratamento coincide com o tempo de descoberta da doença. Além disso, grande parte dos usuários relatou ter realizado a última consulta médica há menos de seis meses da data da entrevista, o que indica regularidade no tratamento. Esses dados justificam a não utilização recente dos serviços de urgência e de internação pela maior parte dos entrevistados.

Com relação à QV, analisando-se todos os domínios do questionário SF-36, observou-se que os usuários das UAPAS de Alfenas-MG manifestaram pouco comprometimento geral, sendo que os domínios apresentaram valores iguais ou superiores a 70 .
Por não ter sido o presente estudo realizado com uma população controle e por não ter um estudo de avaliação da população normal brasileira com o SF-36, os resultados obtidos foram comparados a estudos realizados com doenças crônicas, comumente tratadas nas UAPS.

Os resultados obtidos neste estudo apresentaram valores superiores em todos os domínios do SF-36, quando comparados a um estudo realizado por Halfoun; Mattos; Aguiar (2007), por meio do qual estes avaliaram a QV de uma população hipertensa que não apresentava outras comorbidades, em um Centro Municipal de Saúde do Rio de Janeiro (UAPS). De maneira semelhante, Brito et al. (2008), em seu estudo realizado apenas com portadores de hipertensão arterial dos usuários de UAPS, encontraram domínios com menores scores na "dor" e "estado geral de saúde". Miranda et al (2010), analisando a QV de idosos portadores de diabetes mellitus em UAPS, também verificaram maior comprometimento da QV. Portanto, pode-se inferir que a QV apresenta-se comprometida na presença de patologias associadas.

Neste estudo, verificou-se que o número de patologias associadas foi pequeno, pois $28 \%$ dos entrevistados relataram não apresentar nenhuma doença crônica. Isso pode explicar a melhor QV encontrada. 


\section{Influência sobre a QV}

A influência da localização da UAPS na QV dos pacientes é um aspecto relevante que deve ser levado em consideração no planejamento de estratégias de implementação de unidades de saúde. Neste estudo, observou-se que a Unidade 4 apresentou a menor QV. Tal resultado pode estar associado ao fato de esta unidade estar localizada num bairro periférico e as condições socioeconômicas da maioria da população serem menores que nas outras unidades estudadas. Mastropietro et al. (2010) observaram que a QV está associada, dentre outros fatores, à condição socioeconômica dos pacientes, sendo que baixos índices de renda per capita estariam relacionados com baixos escores de qualidade de vida. Assim, é importante ressaltar que a QV também é afetada pelas condições de moradia, de renda e de ocupação (MACKEIGAN; PATHAK, 1992).

Ao estudar a QV em relação ao sexo, verificou-se que os homens apresentaram escores inferiores aos das mulheres nos domínios vitalidade e saúde mental. Os resultados deste estudo mostraram-se contrários ao estudo de Pimenta et al. (2008), no qual o sexo masculino apresentou um escore maior de pontuação, exceto para o estado geral de saúde e de aspectos emocionais.

Com relação à análise da polifarmácia (uso de 5 ou mais medicamentos) e da QV, observou-se que a polifarmácia influenciou de forma negativa os domínios capacidade funcional, estado geral de saúde e vitalidade. As várias patologias associadas implicam na prescrição de fármacos de diferentes grupos terapêuticos, aumentando assim os riscos de reações adversas e de interações medicamentosas, refletindo diretamente na QV desses pacientes (GALVÃO, 2006).

A faixa etária também foi um fator determinante sobre a $\mathrm{QV}$, visto que os pacientes com idade igual ou superior a 65 anos apresentaram maior comprometimento nos valores dos domínios capacidade funcional e aspectos sociais. Tal fato pode ser justificado pela presença de comorbidades, comuns nessa faixa etária, aumentando as dificuldades desses pacientes em executar atividades corriqueiras do seu cotidiano, levando a uma condição de dependência. Consequentemente, com o declínio na capacidade funcional, outros domínios também podem sofre influências negativas (ROSA et al., 2003). Entretanto, o domínio estado geral de saúde mostrou resultado inferior no grupo de pacientes com idade entre 45 e 65 anos, quando comparado com o grupo de mais de 65 anos.

\section{CONCLUSÃO}

Neste estudo, constatou-se que os pacientes atendidos nas UAPS apresentam, em geral, boa qualidade de vida, que varia de acordo com fatores socioeconômicos. A 
UAPA que apresentou menor condição socioeconômica manifestou maior comprometimento na QV. Em relação ao sexo, houve melhor QV entre as mulheres, que apresentaram maiores escores nos domínios da vitalidade e saúde mental. A presença de doença crônica e a necessidade de um tratamento contínuo por um longo período, a presença de comorbidades, assim como o uso de polifarmácia, constituem outros fatores importantes na determinação da QV dessa população.

Este estudo oferece informações sobre a QV dos usuários das UAPS, instrumento necessário para que os profissionais da equipe de saúde percebam as necessidades da população e atuem de forma a garantir sua qualidade e seus resultados sobre a saúde da população.

\section{REFERÊNCIAS BIBLIOGRÁFICAS}

BRASIL, Ministério da Saúde. (1996). Norma Operacional Básica do Sistema Único de Saúde (NOB-SUS 11/96). Brasília: Autor.

BRASIL, Ministério da Saúde. Guia SUS do Cidadão. Brasília, 2004. Disponível em:http://portal.saude.gov.br/portal/saude/cid adao/default.cfm Acesso em 21 de fevereiro de 2011.

BRITO, D. M. S. et al. Qualidade de vida e percepção da doença entre portadores de hipertensão arterial. Cad. Saúde Pública, v. 24, n.4, p. 933-940, 2008.

CASTRO, M. et al. Qualidade de vida de pacientes com insuficiência renal crônica em hemodiálise avaliada através do instrumento genérico SF-36. Rev. Assoc. Med. Bras., São Paulo, v. 49, n. 3, jul./ago. 2003.
CAVALCANTE, M. A. et al. Qualidade de vida de pacientes hipertensos em tratamento ambulatorial. Arquivos Brasileiros de Cardiologia, São Paulo, v. 89, n. 4, out. 2007.

CICONELLI, R. M. Tradução para o português e validação do questionário genérico de avaliação de qualidade de vida "Medical outcomes study 36-item shortform health survey (SF-36). São Paulo: Universidade Federal de São Paulo/UNIFESP, 1997.

FERREIRA, E. B.; CAVALCANTI, P. P.; NOGUEIRA D. A. Função em codigo R para analisar experimentos em DBC simples, em uma so rodada. In: JORNADA CIENTÍFICA DA UNIVERSIDADE FEDERAL DE ALFENAS-MG, 2., 2009, Alfenas. Resumos... Alfenas: Unifal-MG, 2009.

GALVÃO, C. O idoso polimedicado estratégias para melhorar a prescrição. Rev. Port. Clin. Geral, n. 22, p. 747-752, 2006.

HALFOUN, V.L.R.; MATTOS, D.S.; AGUIAR, O.B. Qualidade de vida e controle da pressão arterial em pacientes de uma unidade primária do município do Rio de Janeiro. Rev. Bras. Med. Fam. e Com., v.3, n.10, p. 82-90, 2007.

IBGE - Instituto Brasileiro de Geografia e Estatística. IBGE Cidades. Disponivel em < http://www.ibge.gov.br/cidadesat/topwindow. htm?1>. Acesso em 02 de novembro de 2011.

MACKEIGAN, L.D.; PATHAK, D.S.

Overview of health-related quality-of-life measures. Am. J. Hosp. Pharm., v. 49, n. 9, p. 2236-2245, 1992.

MASTROPIETRO, A. P. et al. Relação entre renda, trabalho e qualidade de vida de pacientes submetidos ao transplante de medula óssea. Rev. Bras. Hematol. Hemoter., v. 32, n. 2, p. 102-107, 2010. 
MIRANDA, L. P. et al.Qualidade de vida de idosos com diabetes mellitus cadastrados na estratégia saúde da família. R. Min. Educ. Fís., edição especial, n. 5, p. 125-135, 2010.

NOGUEIRA, R. et al. Qualidade de vida dos pacientes portadores de síndrome de StevensJohnson. Arquivos Brasileiros de

Oftalmologia, v. 66, p. 67-70, 2003.

PIMENTA, F. A. P. et al. Avaliação da qualidade de vida de aposentados com a utilização do questionário SF-36. Rev. Assoc.

Med. Bras., São Paulo, v. 54, n. 1, jan./fev. 2008 .

R DEVELOPMENT CORE TEAM. R: A language and environment for statistical computing. R Foundation for Statistical Computing, Vienna, Áustria. 2011.

RIBEIRO, M. C. S. A. et al Perfil sociodemográfico e padrão de utilização de serviços de saúde para usuários e não usuários do SUS - PNAD 2003. Rev. Ciênc. Saúde Coletiva, v. 11, n. 4, p. 1011-1022, 2006.

ROSA, T. E. C. et al. Fatores determinantes da capacidade funcional entre idosos. Rev. Saúde Pública, v. 37, n. 1, p. 40-48, 2003.

SEIDL, E. M. F.; ZANNON, C.M.L.C. Qualidade de vida e saúde: aspectos conceituais e metodológicos. Cad. Saúde Pública, v. 20, n. 2, p.580-588, 2004.

SOARES, D. A. et al. Qualidade de vida de portadores de insuficiência cardíaca. Rev. Acta Paulista de Enfermagem, São Paulo, v. 21, n. 2, 2008.

Sociedade Brasileira de Cardiologia / Sociedade Brasileira de Hipertensão / Sociedade Brasileira de Nefrologia. VI Diretrizes Brasileiras de Hipertensão. Arq Bras Cardiol, v. 95, n. 1 (supl.1), p. 1-51, 2010 .

SOUZA, R.A.; CARVALHO, A.M. Programa de Saúde da Família e qualidade de vida: um olhar da Psicologia. Estudos de Psicologia, v.8, n.3, p. 515-523, 2003.
TRAVASSOS, C.; VIACAVA, F.;

PINHEIRO, R.; BRITO, A. Utilização dos

serviços de saúde no Brasil: gênero, características familiares e condição social.

Revista Panamericana de Saúde Pública. v. 11, n. 5, p. 365-73, 2002.

WARE Jr, J.E.; SHERBOURNE, C.D. The MOS 36-Item Short-Form Health Survey (SF36). I Conceptual framework and item selection. Med. Care, v. 30, n. 6, p. 473-483, 1992. 